Conclusion: The period prevalence of AEs recorded during MMF treatment appeared as high as $71.7 \%$ in our real life setting. Even though we did not record any serious $\mathrm{AE}$, our analysis suggests a close monitoring of this therapy in SSc. Disclosure of Interests: Rossella Chieffo: None declared, Maria Sofia Ciliento: None declared, Veronica Giacco: None declared, GIUSEPPE SCALISE: None declared, Serena Vettori Consultant for: Boehringer Ingelheim Italia SpA DOI: 10.1136/annrheumdis-2019-eular.8203

\section{OP0064 EVIDENCE-BASED CONSENSUS RECOMMENDATIONS FOR THE IDENTIFICATION AND MANAGEMENT OF INTERSTITIAL LUNG DISEASE IN SYSTEMIC SCLEROSIS}

Anna-Maria Hoffmann-Vold ${ }^{1}$, Toby M. Maher ${ }^{2}$, Edward E. Philpot ${ }^{3}$, Ali Ashrafzadeh ${ }^{4}$, Oliver Distler ${ }^{5} .{ }^{1}$ Oslo University Hospital, Department of Rheumatology, Oslo, Norway, ${ }^{2}$ National Heart and Lung Institute, Imperial College London and Interstitial Lung Disease Unit, Royal Brompton Hospital, London, United Kingdom; ${ }^{3}$ Respiratory Center of Excellence, IQVIA, Durham, NC, United States of America; ${ }^{4}$ Rheumatology Center of Excellence, IQVIA, San Diego, CA, United States of America; ${ }^{5}$ University Hospital Zurich, Department of Rheumatology, Zurich, Switzerland

Background: Interstitial lung disease in systemic sclerosis (SSc-ILD) occurs frequently and carries a high burden of morbidity and mortality. To date, there are no existing guidelines for screening, diagnosis and management of SSc-ILD that would aid early recognition and treatment and improve the care of these patients. Objectives: To develop expert consensus recommendations for the identification and management of SSc-ILD.

Methods: Based on the results of a comprehensive systematic literature analysis conducted in line with NICE/CRD and IQWiG guidelines and PRISMA methodology, evidence-based statements on SSc-ILD risk, screening, diagnosis, treatment and follow-up were developed. A modified Delphi process was then used to establish consensus statements for the identification and management of SSc-ILD. Briefly, an expert panel of 27 European-based pulmonologists, rheumatologists and internists with experience in treating SSc-ILD was established. Between July and November 2018, the panel took part in 3 rounds of online surveys, a face-toface discussion and a WebEx meeting to establish consensus-based recommendations for the management of SSc-ILD. Statements were categorised by topic: risk factors (including biomarkers); screening; diagnosis; assessment of severity; treatment initiation; treatment options; disease progression; treatment escalation; other management options. Panellists indicated their level of agreement with proposed statements on a scale of 1 (strong disagreement) to 7 (strong agreement), and consensus was considered achieved when $\geq 80 \%$ either disagreed (score of $1-3$ ) or agreed (score of 5-7) with a statement. Based on panel feedback, statements that did not reach consensus were modified and re-voted in later rounds.

Results: At the close of the Delphi process, the panel agreed on the following:

1. Risk factors: The presence of anti-topoisomerase I antibodies, male gender and diffuse cutaneous SSc all increase risk for ILD

2. Screening: All SSc patients should undergo screening for ILD, using HRCT and lung function testing. Frequency of screening using HRCT should be guided by risk of developing ILD, in combination with clinical symptoms and lung function

3. Diagnosis and assessment of severity: Use of HRCT to diagnose SSc-ILD and assess severity, with supporting findings from lung function testing and clinical assessment, is also recommended

4. Treatment initiation and options: All patients with severe or progressive SSc-ILD should be considered for pharmacotherapy, with mycophenolate mofetil and cyclophosphamide recommended as treatments. Patients not receiving treatment should be followed closely for signs of disease progression

5. Disease progression: Indicators of progression include sustained decline in lung function, worsening of clinical symptoms, and change in extent and/or pattern of fibrosis on HRCT

6. Treatment escalation: Patients with inadequate treatment responses should be considered for treatment escalation. Suitability for lung transplant should be evaluated early, especially for patients diagnosed with advanced disease. Autologous haematopoietic stem cell transplant may be considered in carefully selected patients

Conclusion: These evidence-based expert consensus recommendations, developed using a modified Delphi process, provide important guidance for the identification and management of SSc-ILD.

Acknowledgement: Funding: Boehringer Ingelheim

Disclosure of Interests: Anna-Maria Hoffmann-Vold Grant/research support from: Received research funding or other remuneration from Boehringer Ingelheim, GSK, and Actelion, Consultant for: Received consulting fees or other remuneration from Boehringer Ingelheim, GSK, and Actelion, Speakers bureau: Actelion and Boehringer Ingelheim, Toby M Maher Shareholder of: Has stock options or bond holdings in a for-profit corporation in Apellis, Grant/research support from: Received funds from BI advisory board participation and conference travel. Received research funding and/or consulting fees or other remuneration from GSK, UCB, AstraZeneca, Roche, Bayer, Biogen Idec, Cipla, Prometic, and Sanumed, Consultant for: Toby Maher has received consultancy or speakers fees from Apellis, AstraZeneca, Bayer, Biogen Idec, Boehringer Ingelheim, Galapagos, GlaxoSmithKline R\&D, Indalo, Pliant, ProMetic, Roche, Samumed, and UCB; and has received consultancy fees from Galecto, Edward E Philpot Employee of: Employee of IQVIA, Ali Ashrafzadeh Employee of: Employee of IQVIA, Oliver Distler Grant/research support from: Prof. Distler received research funding from Actelion, Bayer, Boehringer Ingelheim and Mitsubishi Tanabe to investigate potential treatments of scleroderma and its complications, Consultant for: Prof. Distler has/had consultancy relationship within the last 3 years with Actelion, AnaMar, Bayer, Boehringer Ingelheim, ChemomAb, espeRare foundation, Genentech/Roche, GSK, Inventiva, Italfarmaco, iQvia, Lilly, medac, Medlmmune, Mitsubishi Tanabe Pharma, Pharmacyclics, Novartis, Pfizer, Sanofi, Serodapharm and UCB in the area of potential treatments of scleroderma and its complications. In addition, he had/has consultancy relationship within the last 3 years with A. Menarini, Amgen, Abbvie, GSK, Mepha, MSD, Pfizer and UCB in the field of arthritides and related disorders DOI: 10.1136/annrheumdis-2019-eular.3225

\section{OP0065 SCLEROSIS (VEDOSS) PROJECT: PREDICTORS TO DEVELOP DEFINITE DISEASE FROM AN INTERNATIONAL MULTICENTRE STUDY}

Silvia Bellando Randone ${ }^{1}$, Gemma Lepri ${ }^{1}$, Dorte Husher ${ }^{2}$, Tunde Minier ${ }^{3}$, Serena Guiducci ${ }^{1}$, Cosimo Bruni ${ }^{1}$, László Czirják ${ }^{4}$, Maurizio Cutolo ${ }^{5}$

Vanessa Smith ${ }^{6}$, Jérôme Avouac ${ }^{7}$, Daniel Furst ${ }^{8}$, Yannick Allanore ${ }^{9}$,

Oliver Distler ${ }^{10}$, Marco Matucci-Cerinic ${ }^{1}$, on behalf of VEDOSS co-workers.

${ }^{1}$ Careggi University Hospital, Florence, Italy; ${ }^{2}$ Department of Rheumatology and Clinical Immunology Charité University Hospital, Berlin, Germany, ${ }^{3}$ Department of Immunology and Rheumatology, University of Pécs, Pécs, Hungary; ${ }^{4}$ Department of Immunology and Rheumatology, Pécs, Hungary; ${ }^{5}$ IRCCS San Martino Polyclinic Hospital, University Of Genoa, Genoa, Italy; ${ }^{6}$ University of Ghent, Ghent, Belgium; ${ }^{7}$ Cochin Hopital, Paris, Italy; ${ }^{8}$ UCLA, Los Angeles, United States of America; ${ }^{9}$ Cochin Hospital, Paris, France; ${ }^{10}$ University Hospital Zurich, Zurich, Swaziland

Background: Early identification of patients is of key importance for the management and treatment of inflammatory rheumatic diseases.

Objectives: The aim of the VEDOSS project (1) is to determine through an at-risk population the predictive factors for the progression toward a definite systemic sclerosis (SSc).

Methods: VEDOSS investigators prospectively recruited patients with Raynaud phenomenon (RP), with or without anti-nuclear antibodies (ANA)for this longitudinal, observational study. Fulfilling the 2013 classification criteria at baseline was an exclusion criterion. Patients with primary Raynaud syndrome were recruited as controls. Patients had an annual assessment according to EUSTAR standards to determine organ involvement and severity. The endpoint was defined as fulfilment of the 2013 classification criteria. The time to fulfilling 2013 classification criteria was evaluated with Kaplan-Meier analysis, and predictors of evolution were determined by univariate and multivariate Cox regression.

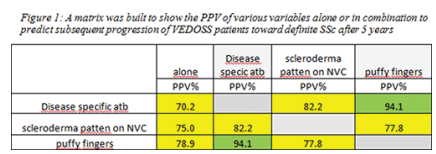

Results: 735 patients with RP were recruited into the study. The sample is distributed as follows: i) 237 patients (143 with follow up) RP/ANA negative (ANA $/ p R P$ as the control group, ii) 498 patients ( 401 with follow up) RP/ANA positive (ANA ${ }^{+}$ pRP)): 87 had puffy fingers (PF), 199 had anti-centromere antibodies (atb) positive, 45 had anti-topoisomerase I atb positive and 182 had nailfold videocapillaroscopy (NVC) abnormalities at baseline. Out of 401 ANA $^{+} / p R P$ patients, $7.4 \%$ within 1 year, $29.3 \%$ within 3 and $44.1 \%$ within 5 years satisfied the 2013 classification criteria. Out of the $143 \mathrm{ANA}^{-} / \mathrm{pRP}$ patients, none $(0 \%)$ within 1 year, $4.6 \%$ within 3 years, and $4,6 \%$ within 5 years satisfied SSc criteria. After adjustment for age, the following baseline parameters were identified as independent predictors for progression into definite SSc by multivariate analysis: puffy fingers (OR=3.4 [2.0;5.6]), anti-centromere atb $(\mathrm{OR}=2.6[1.6 ; 4.1])$ and anti-topoisomerase 1 atb $(\mathrm{OR}=3.1$ [1.6;5.8]), and NVC abnormalities $(\mathrm{OR}=1.9[1.3 ; 2.9])$ The presence of PF had a positive predictive value (PPV) of $79 \%$ and combination of PF + specific auto-antibodies showed 94\% PPV to satisfy ACR/EULAR 2013 criteria within 5 years (figure 1).

Conclusion: the data show that patients with very early SSc develop definite, classification criteria fulfilling SSc within 5 years of follow up. The VEDOSS study 